\title{
Estimation des calories supplémentaires liées à la consommation d'alcool comme facteur potentiellement négligé de l'obésité chez les jeunes
}

\author{
Kate Battista, M. Math.; Scott T. Leatherdale, Ph. D.
}

Cet article a fait l'objet d'une évaluation par les pairs.

Diffuser cet article sur Twitter

\begin{abstract}
Résumé
Introduction : Les taux d'obésité chez les jeunes ne cessent d'augmenter au Canada. Dans cette étude, nous avons réalisé des estimations conservatrices des calories excédentaires susceptibles de découler de la consommation d'alcool en fonction des habitudes de consommation courantes chez les jeunes Canadiens, afin de déterminer si la consommation d'alcool devrait être prise en compte dans les futures stratégies de prévention de l'obésité.
\end{abstract}

Méthodologie : Des données portant sur 10144 élèves de $12^{\mathrm{e}}$ année ayant participé à l'étude COMPASS (2013-2014) ont été utilisées pour estimer l'apport calorique annuel provenant de la consommation d'alcool. Pour effectuer nos estimations, nous avons classé les boissons alcoolisées en trois catégories, en fonction de leur teneur moyenne en calories (coolers à base de vodka; bière $5 \%$; bière $4 \%$, vin et spiritueux) et de la fréquence de consommation et d'abus occasionnel d'alcool (ou hyperalcoolisation rapide).

Résultats : Les résultats ont indiqué un apport calorique potentiel élevé chez les élèves qui s'adonnaient aux abus occasionnels d'alcool, ainsi qu'une forte variabilité dans les estimations de l'apport calorique en fonction des habitudes de consommation courantes associées aux différents types de boisson. Par exemple, 27,2 \% des élèves s'adonnaient à un abus occasionnel d'alcool une fois par mois, ce qui représente une consommation de 6000 à 13200 calories par année (soit 0,78 à 1,71 kg de gras). Pour les 4,9\% d'élèves qui s'adonnaient à un abus occasionnel d'alcool deux fois par semaine, le nombre de calories consommées en une année se situerait entre 52000 et 114400 (soit 6,74 à 14,83 kg de gras).

Conclusion : Les recommandations en vigueur pour la prévention de l'obésité chez les jeunes ne tiennent généralement pas compte de la consommation d'alcool. Étant donné à la fois les taux élevés de consommation d'alcool et d'abus occasionnels d'alcool fréquents chez les jeunes mis en lumière dans cette étude et la grande quantité de calories que contiennent les boissons alcoolisées, tenir compte de la consommation d'alcool dans les efforts de prévention de l'obésité chez les jeunes serait pertinent.

Mots-clés : consommation d'alcool, abus occasionnel d'alcool, stratégies de prévention, gain de poids, calories supplémentaires, apport énergétique, jeunes

\section{Introduction}

Le surplus de poids chez les jeunes constitue un problème de santé publique, l'obésité juvénile étant associée à des effets immédiats et à long terme variés sur la santé, notamment le diabète de type 2 et les maladies cardiaques ${ }^{1}$. D'après les données de l'Enquête canadienne sur les mesures de la santé de 2009 à 2011, chez les Canadiens de 12 à 17 ans, 29,6 \% des garçons et $30,5 \%$ des filles présenteraint un surplus de poids ou seraient obèses ${ }^{2}$. La prévalence de l'embonpoint et de

\section{Points saillants}

- L'obésité chez les jeunes est un problème de santé publique préoccupant. Les lignes directrices actuelles en matière de prévention de l'obésité sont principalement axées sur la consommation d'aliments et de boissons sucrées, et pourraient négliger les effets potentiels des calories consommées avec les boissons alcoolisées.

- La consommation fréquente et excessive d'alcool est courante chez les jeunes au Canada. Les calories fournies par l'alcool sont dépourvues de valeur nutritive et s'ajoutent à la consommation alimentaire.

- La présente étude fournit des estimations conservatrices du nombre de calories consommées sous forme d'alcool en fonction des habitudes de consommation observées chez des jeunes de $12^{\mathrm{e}}$ année de l'Ontario et de l'Alberta.

- Des taux élevés de consommation d'alcool et d'abus occasionnels d'alcool fréquents chez les jeunes constituent un apport important en calories sur l'année.

l'obésité ayant considérablement augmenté au cours des deux dernières décennies $^{3}$, diverses solutions et stratégies de prévention de l'obésité ont été élaborées afin de contrer ce problème grandissant, que ce soit des interventions à l'échelle de la population ${ }^{4,5}$ ou des interventions cliniques $^{6,7}$. Elles visent généralement l'atteinte de l'équilibre énergétique par l'activité physique et l'amélioration de l'alimentation.

Rattachement des auteurs :

École de santé publique et de systèmes de soins de santé, Université de Waterloo, Waterloo (Ontario), Canada

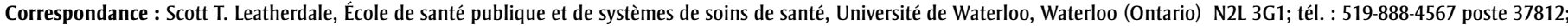
téléc. : 519-746-2510; courriel : sleather@uwaterloo.ca 
Dans le domaine de la diététique, les calories supplémentaires provenant de la consommation de boissons sucrées (p. ex. jus de fruits et boissons gazeuses) font l'objet d'une attention accrue depuis quelque temps, en raison de leur lien avec l'obésité ${ }^{8}$. Dans un rapport publié en 2016 par le Comité sénatorial permanent des affaires sociales, des sciences et de la technologie, L'obésité au Canada : une approche pansociétale pour un Canada en meilleure santé, on a recommandé au gouvernement fédéral de mettre en place une taxe sur les boissons sucrées ${ }^{9}$. Or, alors que les intervenants travaillent à élaborer des stratégies pour freiner la consommation de boissons sucrées chez les jeunes, très peu d'attention est accordée à l'impact potentiel des calories excédentaires provenant de la consommation de boissons alcoolisées. Des données probantes indiquent pourtant un lien entre la consommation d'alcool et le risque de gain de poids ou d'obésité chez les jeunes ${ }^{10-13}$, en particulier chez les filles $^{12-13}$. Non seulement l'alcool peut constituer une importante source de calories (7,1 kcal/g) n'ayant pas ou très peu de valeur nutritive mais il s'ajoute aussi généralement à l'apport calorique ${ }^{14-15}$. L'alcool est également susceptible de représenter un facteur de risque indirect de l'obésité lorsqu'il est associé à une modification de l'alimentation, par exemple une augmentation à la quantité d'aliments ingérés après la consommation d'alcool ${ }^{15}$, ou à des troubles alimentaires tels que la frénésie alimentaire ${ }^{16}$

Les discussions sur la consommation d'alcool et le gain de poids portent généralement sur les populations adultes, et il existe peu de recherches sur les liens entre la consommation d'alcool et le gain de poids chez les jeunes. La consommation fréquente et excessive d'alcool est très répandue chez les jeunes Canadiens ; dans une étude menée en 2011, 25,5\% des élèves de la $9^{\mathrm{e}}$ à la $12^{\mathrm{e}}$ année s'adonnaient à des abus occasionnels d'alcool ${ }^{17}$. En outre, la consommation maximale d'alcool à un jeune âge a été associée à une consommation excessive d'alcool et à l'obésité à l'âge adulte ${ }^{18}$. Les jeunes devraient donc être considérés comme étant une population potentiellement à risque.

L'étude de référence COMPASS (Cohort Study of Obesity, Marijuana Use, Physical Activity, Alcohol Use, Smoking and Sedentary Behaviours) est une étude de cohorte menée auprès d'élèves du secondaire de l'Ontario et de l'Alberta qui vise à mesurer l'impact des décisions en matière de politique sur le comportement des élèves sur le plan de la santé ${ }^{19}$. Le sondage annuel rempli par les élèves dans le cadre de cette étude contient des questions sur la fréquence de consommation d'alcool et d'abus occasionnels d'alcool. Notre étude utilise des données secondaires tirée de l'étude COMPASS pour réaliser des estimations conservatrices du nombre total de calories potentiellement consommées avec l'alcool, selon différentes habitudes de consommation d'alcool courantes chez les jeunes ayant participé à l'étude. Compte tenu du grand nombre de publications traitant des problèmes liés aux abus occasionnels d'alcool pendant l'adolescence, notre article ne traite pas des risques pour la santé associés à la consommation d'alcool mais plutôt du problème que peut constituer l'apport en calories supplémentaires attribuable à la consommation d'alcool.

\section{Méthodologie}

\section{Conception}

Notre étude est fondée sur des données secondaires transversales de l'année 2 (2013-2014) de l'étude de référence COMPASS $^{19}$, ce qui nous a permis de disposer d'un échantillon composé de davantage d'élèves de $12^{\mathrm{e}}$ année que si nous avions choisi l'année 1 , pour laquelle nous disposions d'un plus petit nombre d'écoles. Les données ont été recueillies à l'aide du questionnaire de l'élève COMPASS accompagnées de procédures d'information active et de consentement passif. Toutes ces procédures ont été approuvées par le bureau d'éthique de la recherche de l'Université de Waterloo et par les conseils scolaires concernés. Une description complète des méthodes utilisées dans l'étude est disponible en version imprimée ${ }^{19}$ et en version en ligne (www.compass.uwaterloo .ca).

\section{Paramètres mesurés}

La présente étude est fondée sur des données secondaires provenant des questionnaires remplis par les élèves. Le questionnaire COMPASS permet de recueillir des données individuelles sur les élèves en matière de comportements (consommation d'alcool, tabagisme, obésité, activité physique, comportements alimentaires, etc.) et à propos des corrélats de ces comportements et des caractéristiques individuelles. Dans chaque école, le questionnaire a été rempli par tous les élèves pendant les heures de classe. Les questions étaient basées sur les normes nationales et les lignes directrices nationales en vigueur en matière de santé publique disponibles ailleurs ${ }^{19}$.

\section{Participants}

Pour l'année 2, 89 écoles (79 de l'Ontario et 10 de l'Alberta) ayant approuvé l'utilisation de protocoles d'information active et de consentement parental passif ont été invitées à participer à l'étude COMPASS. Les élèves pouvaient à tout moment interrompre leur participation. Le taux de participation à l'ensemble de l'étude a été de 78,2\%, le principal motif de non-participation étant l'absence lors de la collecte des données. Notre article s'appuie sur les données de l'année 2, qui ont été recueillies auprès de 10144 élèves (4 927 filles et 5217 garçons) de $12^{\mathrm{e}}$ année. Des recherches antérieures ont révélé que les répondants de $12^{\mathrm{e}}$ année (en général âgés de 16 à 18 ans) étaient ceux qui présentaient les taux les plus élevés en matière de consommation d'alcool ${ }^{17}$. Conformément aux protocoles de collecte de données de l'étude COMPASS, les élèves ont rempli les questionnaires pendant les heures de classe, le jour prévu par leur école pour cette activité.

\section{Mesures}

Les deux questions portant sur la consommation d'alcool (fréquence de consommation et fréquence des abus occasionnels d'alcool) qui figuraient dans le questionnaire ont été tirées d'outils de surveillances nationaux déjà utilisés pour les jeunes au Canada $^{20}$. Dans ce questionnaire, un verre correspondait à " une bouteille ou une canette de bière de taille standard, un verre de bière en fût, une coupe de vin, une bouteille de cooler, un verre de spiritueux ou un cocktail » respectant les portions définies par le Centre canadien de lutte contre les toxicomanies (CCLT) ${ }^{21}$.

\section{Fréquence de la consommation d'alcool}

Pour évaluer la fréquence de la consommation d'alcool, on a posé la question suivante " $\mathrm{Au}$ cours des 12 derniers mois, combien de fois as-tu consommé plus qu'une gorgée d'alcool? ». Les choix de réponse étaient : "Je n'ai jamais consommé d'alcool ", "Je n'ai pas consommé d'alcool au cours des 12 derniers mois", " Je n'ai pris qu'une gorgée d'alcool ", " Moins d'une fois par mois », « Une fois 
par mois », « 2 ou 3 fois par mois », " Une fois par semaine », « 2 ou 3 fois par semaine », " 4 à 6 fois par semaine ", et " Tous les jours ». Les catégories de réponses ont été combinées de façon à créer cinq fréquences de consommation d'alcool, soit " Aucune consommation d'alcool ", " Moins d'une fois par mois ", " 1 à 3 fois par mois ", " 1 à 3 fois par semaine », et " 4 fois ou plus par semaine ». Le taux de réponse à cette question a été de $97,92 \%$, et les élèves n'ayant pas répondu à la question n'ont pas été pris en compte dans les analyses.

\section{Fréquence des abus occasionnels d'alcool}

La mesure de la fréquence des abus occasionnels d'alcool fournit des renseignements sur les niveaux de consommation, sur la base de cinq verres par occasion. La question suivante était posée aux répondants : «Au cours des 12 derniers mois, combien de fois as-tu pris 5 verres ou plus à une même occasion? ". Les choix de réponse étaient "Je ne l'ai jamais fait ", "Je n'ai pas pris 5 verres ou plus à une même occasion au cours des 12 derniers mois ", " Moins d'une fois par mois ", "Une fois par mois ", " 2 à 3 fois par mois ", "Une fois par semaine ", " 2 à 5 fois par semaine ", et "Tous les jours ou presque tous les jours ». Les catégories de réponses ont été combinées de façon à créer cinq fréquences d'abus occasionnel d'alcool, soit " Aucun abus occasionnel d'alcool ", " Moins d'une fois par mois », " 1 à 3 fois par mois ", "Une fois par semaine », et " 2 fois ou plus par semaine ». Le taux de réponse à cette question était de 97,96\%, et les élèves n'ayant pas répondu à la question n'ont pas été pris en compte dans les analyses. Bien que la question visant à mesurer la fréquence des abus occasionnels d'alcool ne respecte pas les lignes directrices sur la consommation d'alcool à faible risque en ce qui concerne l'hyperalcoolisation rapide chez les femmes (quatre verres ou plus en une même occasion) ${ }^{21}$, elle cadre avec les mesures des outils de surveillance nationaux utilisés auprès des jeunes ${ }^{20}$ et fournit une estimation plus conservatrice des abus occasionnels d'alcool chez les femmes.

\section{Analyses}

Nous avons calculé la fréquence de consommation d'alcool et d'abus occasionnels d'alcool par sexe et nous avons utilisé des tests du chi carré pour comparer les habitudes de consommation entre les sexes. Nous avons consulté le Fichier canadien sur les éléments nutritifs (FCÉN) pour connaître la teneur en calories de différents types d'alcool, puis nous avons calculé la teneur pour un verre standard en utilisant les formats établis par le CCLT ${ }^{21}$. Douze boissons alcoolisées, assorties de leur apport en calories, ont été répertoriées dans la base de données. Nous avons calculé l'apport en calories d'un verre standard en fonction des définitions du CCLT : un verre correspond à une bouteille de bière ou de cooler (341 $\mathrm{ml}$ [12 oz]), un verre de spiritueux (43 ml [1,5 oz]), ou un verre de vin (142 $\mathrm{ml}$ [5 oz]). En utilisant la liste du FCÉN, nous avons regroupé les boissons selon le nombre de calories approximatif contenu dans une portion standard, pour obtenir trois catégories représentatives : teneur élevée en calories, teneur moyenne en calories, faible teneur en calories. Les coolers à la vodka ont une teneur élevée en calories, avec 220 calories par portion standard. La bière ordinaire (5\% d'alcool par volume) a une teneur moyenne en calories, soit 140 calories par portion standard. La bière légère (4\% d'alcool par volume), le vin de table (rouge ou blanc) et les spiritueux (gin, rhum, vodka et whisky) contiennent tous environ 100 calories par portion standard. Nous avons estimé le nombre de calories consommées sous forme d'alcool par les élèves, pour différentes catégories génériques de boissons alcoolisées classées d'après leur valeur calorigène moyenne [cooler à la vodka, bière $(5 \%)$, bière $(4 \%)$, vins et spiritueux] et pour les cinq fréquences établies de consommation d'alcool et d'abus occasionnels d'alcool. Nous avons converti le nombre total de calories consommées sur une période d'un an en poids équivalent, en calculant une kilogramme par 7716 calories $^{23}$. Toutes les analyses statistiques ont été réalisées à l'aide du progiciel SAS, version 9.4 (SAS Institute Inc., Cary, Caroline du Nord, États-Unis). Un seuil de signification de $p<0,05$ a été utilisé dans tous les tests statistiques.

\section{Résultats}

Le tableau 1 présente la fréquence de consommation d'alcool et d'abus occasionnels d'alcool chez les répondants de l'année 2 , par sexe. Sur l'ensemble du groupe, 53,6 \% des élèves ont déclaré consommer de l'alcool au moins une fois par mois, tandis que $18,7 \%$ ont déclaré en consommer au moins une fois par semaine. La proportion de jeunes ne consommant pas d'alcool était sensiblement la même chez les deux sexes $(24,1 \%$ pour les garçons et $24,4 \%$ pour les filles, $p=0,774)$, mais les tests du chi carré $\left(\chi^{2}\right)$ ont révélé des habitudes de consommation très différentes chez les filles et les garçons $\left(\chi^{2}=235,19\right.$, degrés de

\section{TABLEAU 1}

Fréquence de la consommation d'alcool et des abus occasionnels d'alcool selon le sexe, chez les élèves de $12^{\mathrm{e}}$ année ayant participé à l'année 2 (2013-2014) de l'étude COMPASS, Ontario et Alberta, Canada

\begin{tabular}{|c|c|c|c|c|c|c|}
\hline & \multicolumn{2}{|c|}{ Total } & \multicolumn{2}{|c|}{ Garçons } & \multicolumn{2}{|c|}{ Filles } \\
\hline & (n) & $\%$ & (n) & $\%$ & (n) & $\%$ \\
\hline \multicolumn{7}{|l|}{ Consommation d'alcool } \\
\hline $\begin{array}{l}\text { Aucune consommation } \\
\text { d'alcool }\end{array}$ & 2408 & 24,3 & 1222 & 24,1 & 1186 & 24,4 \\
\hline Moins d'une fois par mois & 2193 & 22,2 & 932 & 18,5 & 1261 & 25,9 \\
\hline 1 à 3 fois par mois & 3467 & 34,9 & 1704 & 33,6 & 1763 & 36,2 \\
\hline 1 à 3 fois par semaine & 1564 & 15,7 & 973 & 19,2 & 591 & 12,1 \\
\hline 4 fois et plus par semaine & 301 & 3,0 & 235 & 4,6 & 66 & 1,4 \\
\hline Total & 9933 & $100,1^{\mathrm{a}}$ & 5066 & 100,0 & 4867 & 100,0 \\
\hline \multicolumn{7}{|l|}{ Abus occasionnels d'alcool } \\
\hline $\begin{array}{l}\text { Aucune abus occasionnel } \\
\text { d'alcool }\end{array}$ & 3900 & 39,2 & 1860 & 36,7 & 2040 & 41,9 \\
\hline Moins d'une fois par mois & 2205 & 22,2 & 1020 & 20,1 & 1185 & 24,4 \\
\hline 1 à 3 fois par mois & 2699 & 27,2 & 1412 & 27,8 & 1287 & 26,5 \\
\hline 1 fois par semaine & 649 & 6,5 & 421 & 8,3 & 228 & 4,7 \\
\hline 2 fois et plus par semaine & 484 & 4,9 & 360 & 7,1 & 124 & 2,5 \\
\hline Total & 9937 & 100,0 & 5073 & 100,0 & 4864 & 100,0 \\
\hline
\end{tabular}

${ }^{a}$ Les chiffres ayant été arrondis, leur somme ne correspond pas à 100,0. 
liberté $[\mathrm{dl}]=4, p<0,001)$, ces derniers consommant de l'alcool plus fréquemment que leurs homologues de sexe féminin. Dans l'ensemble, 38,6 \% des élèves ont déclaré s'adonner aux abus occasionnels d'alcool au moins une fois par mois, tandis que $11,4 \%$ ont déclaré le faire au moins une fois par semaine. Les tests du chi carré ont révélé des tendances très différentes entre les sexes à ce chapitre $\left(\chi^{2}=194,60\right.$, $\mathrm{dl}=4, p<0,001$ ), les garçons s'adonnant plus fréquemment aux abus occasionnels d'alcool que les filles. Les garçons étaient deux fois plus susceptibles que les filles de s'adonner aux abus occasionnels d'alcool au moins une fois par semaine $(15,4 \%$ et $7,2 \%$, respectivement), et presque trois fois plus susceptibles que celles-ci de le faire deux ou trois fois par semaine $(7,1 \%$ et $2,5 \%$, respectivement).

\section{Estimation de l'apport calorique}

Le tableau 2 présente des estimations du nombre total de calories consommées sur une période d'un an par les élèves ayant déclaré boire de l'alcool au moins une fois par mois, selon la fréquence de consommation et le type de boisson. Les résultats indiquent une forte variabilité dans le total de calories consommées selon le type de boisson et la fréquence de consommation. Par exemple, les 15,7 \% d'élèves qui ont déclaré boire de l'alcool une fois par semaine auraient consommé entre 5200 et 11440 calories en un an (soit 0,67 à 1,48 kg de gras), en supposant qu'ils ne prenaient qu'une seule consommation à chaque occasion. Pour les 3,0 \% d'élèves $(4,6 \%$ des garçons, $1,4 \%$ des filles), qui disaient boire de l'alcool quatre fois par semaine, le nombre de calories consommées en un an se situerait entre 20800 et 45760 (soit 2,70 à 5,93 kg de gras). La figure 1 montre les estimations du nombre de calories consommées, en supposant un seul verre par occasion par type de boisson, et le pourcentage d'élèves qui se situent dans chaque plage d'estimation.

TABLEAU 2

Calories supplémentaires associées à la consommation d'alcool et aux abus occasionnels d'alcool sur une période d'un an, selon le type de boisson et la fréquence de consommation, chez les élèves de $12^{\mathrm{e}}$ année ayant participé à l'année 2 (2013-2014) de l'étude COMPASS, Ontario et Alberta, Canada

\begin{tabular}{|c|c|c|c|c|}
\hline & $\begin{array}{l}\% \text { des } \\
\text { élèves }\end{array}$ & Type de boisson & $\begin{array}{c}\text { Calories } \\
\text { consommées }\end{array}$ & $\begin{array}{c}\text { Équivalent en } \\
\text { kilogrammes de gras } \\
\text { des calories fournies } \\
\text { par l'alcool }\end{array}$ \\
\hline \multicolumn{5}{|c|}{ Fréquence de la consommation d'alcool } \\
\hline Une fois par mois & 34,9 & $\begin{array}{l}\text { Cooler à la vodka } \\
\text { Bière ( } 5 \text { \%) } \\
\text { Bière ( } 4 \text { \%), vin, spiritueux }\end{array}$ & $\begin{array}{l}2640 \\
1680 \\
1200\end{array}$ & $\begin{array}{l}0,34 \\
0,22 \\
0,16\end{array}$ \\
\hline Une fois par semaine & 15,7 & $\begin{array}{l}\text { Cooler à la vodka } \\
\text { Bière ( } 5 \text { \%) } \\
\text { Bière (4 \%), vin, spiritueux }\end{array}$ & $\begin{array}{r}11440 \\
7280 \\
5200\end{array}$ & $\begin{array}{l}1,48 \\
0,94 \\
0,67\end{array}$ \\
\hline Quatre fois par semaine & 3,0 & $\begin{array}{l}\text { Cooler à la vodka } \\
\text { Bière ( } 5 \text { \%) } \\
\text { Bière (4 \%), vin, spiritueux }\end{array}$ & $\begin{array}{l}45760 \\
29120 \\
20800\end{array}$ & $\begin{array}{l}5,93 \\
3,77 \\
2,70\end{array}$ \\
\hline Fréquence des abus occa & ionnels & alcool & & \\
\hline Une fois par mois & 27,2 & $\begin{array}{l}\text { Cooler à la vodka } \\
\text { Bière }(5 \%) \\
\text { Bière }(4 \%) \text {, vin, spiritueux }\end{array}$ & $\begin{array}{r}13200 \\
8400 \\
6000\end{array}$ & $\begin{array}{l}1,71 \\
1,09 \\
0,78\end{array}$ \\
\hline Une fois par semaine & 6,5 & $\begin{array}{l}\text { Cooler à la vodka } \\
\text { Bière }(5 \%) \\
\text { Bière }(4 \%) \text {, vin, spiritueux }\end{array}$ & $\begin{array}{l}57200 \\
36400 \\
26000\end{array}$ & $\begin{array}{l}7,41 \\
4,72 \\
3,37\end{array}$ \\
\hline Deux fois par semaine & 4,9 & $\begin{array}{l}\text { Cooler à la vodka } \\
\text { Bière }(5 \%) \\
\text { Bière }(4 \%) \text {, vin, spiritueux }\end{array}$ & $\begin{array}{r}114400 \\
72800 \\
52000\end{array}$ & $\begin{array}{r}14,83 \\
9,43 \\
6,74\end{array}$ \\
\hline
\end{tabular}

Les 27,2 \% d'élèves qui ont déclaré s'adonner aux abus occasionnels d'alcool une fois par mois, quant à eux, auraient consommé entre 6000 et 13200 calories sur une période d'un an (soit 0,78 à 1,71 kg de gras; tableau 2), en supposant cinq verres d'alcool par occasion. Pour les $4,9 \%$ d'élèves $(7,1 \%$ des garçons, $2,5 \%$ des filles) qui ont déclaré le faire deux fois par semaine, le nombre total de calories consommées sur une période d'un an serait de 52000 à 114400 (soit 6,74 à $14,83 \mathrm{~kg}$ de gras). La figure 2 montre les estimations du nombre de calories consommées, en supposant cinq verres par occasion, par type de boisson, et le pourcentage d'élèves qui se situent dans chaque plage d'estimation.

\section{Analyse}

L'obésité chez les jeunes est un problème de santé publique, d'autant plus que l'obésité à l'adolescence peut avoir des conséquences néfastes sur la santé à l'âge adulte $^{24}$. Le risque d'obésité chez les jeunes dépend d'une variété de facteurs, notamment de facteurs liés au mode de vie tels que l'apport alimentaire et l'activité physique $^{6,11}$. Bien que la consommation d'alcool ne représente qu'une partie du problème, les facteurs de risque modifiables sont souvent liés entre $\mathrm{eux}^{25}$, et ces liens devraient être pris en compte dans l'élaboration d'interventions en santé publique ${ }^{26}$. Les recommandations actuelles pour la prévention de l'obésité chez les jeunes ne tiennent généralement pas compte de la consommation d'alcool ${ }^{4-7,9}$. Par exemple, dans le dernier rapport sénatorial sur l'obésité au Canada9 , aucune des 21 recommandations visant à prévenir l'obésité chez les jeunes Canadiens ne faisait mention, ni ne tenait compte, de l'incidence de la consommation d'alcool. Dans notre étude, la majorité des jeunes de notre échantillon ont déclaré consommer de l'alcool, et nos estimations montrent clairement que même une consommation modérée d'alcool peut accroître de façon importante le nombre de calories consommées en un an. Chez les jeunes les plus à risque ayant indiqué consommer de l'alcool fréquemment et en grande quantité (plus de 1 élève sur 10 dans notre échantillon), la quantité totale de calories absorbées est encore plus préoccupant. Compte tenu des éléments probants présentés dans cet article, nous croyons que la consommation d'alcool est un facteur qui contribue de façon significative à l'embonpoint et à l'obésité chez les jeunes, et qui devrait être pris en compte 
FIGURE 1

Projections du nombre de calories supplémentaires consommées par les élèves sur une période d'un an, sur la base d'un verre d'alcool par occasion, selon le type de boisson, chez les élèves de $12^{\mathrm{e}}$ année ayant participé à l'année 2 (2013-2014) de l'étude COMPASS, Ontario et Alberta, Canada
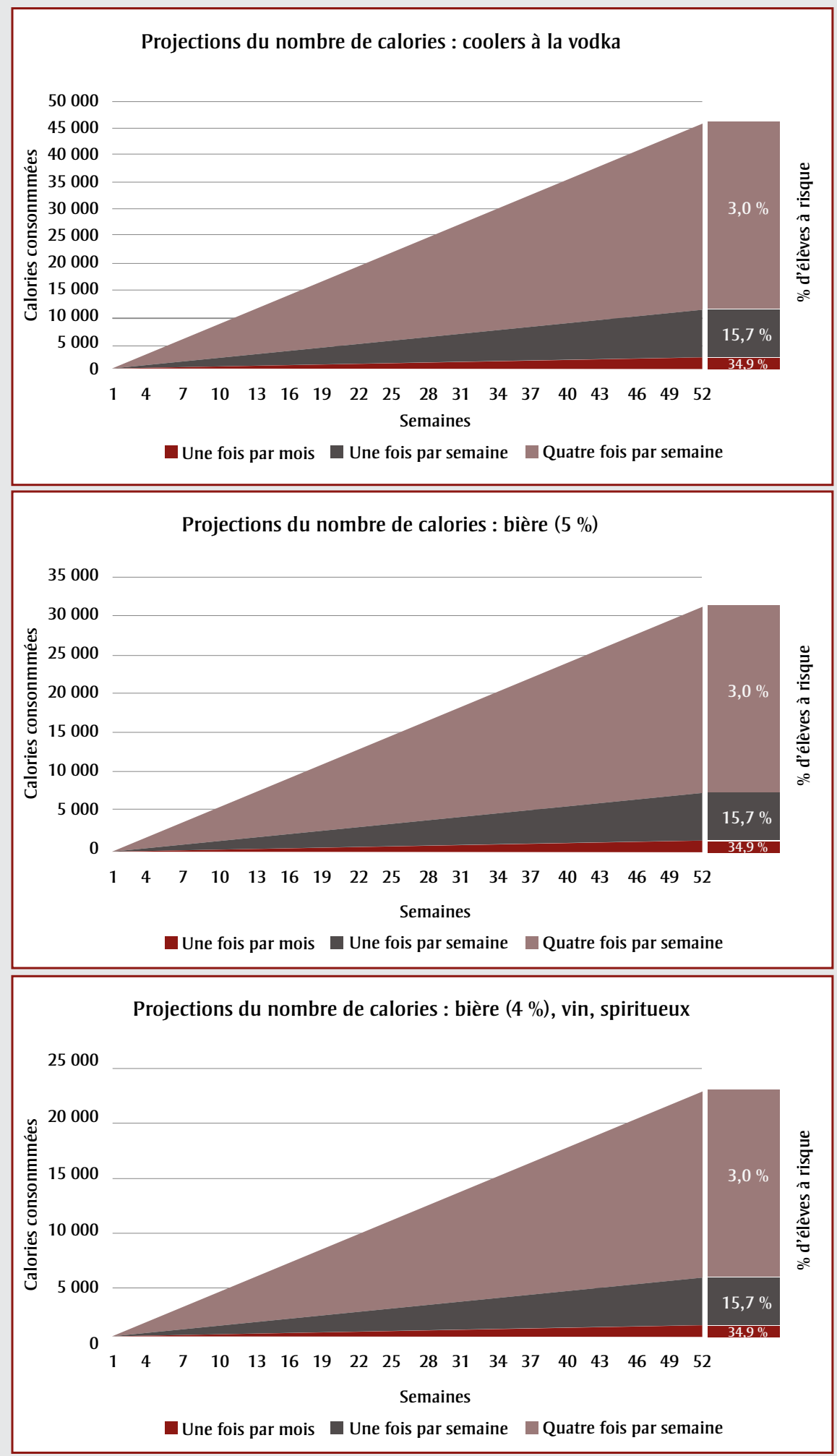

dans l'élaboration des stratégies de prévention de l'obésité.

\section{Forces et limites}

Avec cet article, nous sommes les premiers à fournir des estimations très conservatrices du nombre de calories consommées par les élèves en une seule occasion, sur la base d'une ou cinq consommations par occasion, d'après un vaste échantillon de jeunes Canadiens. Cependant, selon les données du Sondage sur la consommation de drogues et la santé des élèves de l'Ontario (SCDSEO), les jeunes qui boivent déclarent généralement prendre deux consommations ou plus par occasion, plutôt qu'une seule ${ }^{27}$. De nouvelles données probantes semblent également indiquer que lors des abus occasionnels d'alcool, les jeunes prennent souvent 10 à 15 consommations, plutôt que cinq ${ }^{28}$. Bien que les estimations que nous présentons soient très conservatrices, le nombre de calories consommées sous forme d'alcool est relativement élevé pour divers profils de consommation d'alcool qui sont courants chez les jeunes Canadiens ${ }^{17,29}$.

Ces données comportent certaines limites. Le type de boisson constitue un facteur important du nombre total de calories estimé, mais nous ne disposions d'aucune donnée sur le type de boissons alcoolisées habituellement consommées par les élèves de l'échantillon. Les données relatives aux calories contenues dans les cocktails n'étaient pas prises en compte dans l'analyse, en raison de la variabilité dans les types de mélanges et les proportions. Par conséquent, nos estimations ne tenaient pas compte des calories supplémentaires provenant des autres constituants des mélanges (c.-à-d. boissons gazeuses ou jus). Bon nombre des cocktails les plus courants peuvent contenir jusqu'à 490 calories par verre ${ }^{30}$, ce qui montre une fois de plus que nos estimations sont très conservatrices. En outre, les estimations obtenues sont fondées sur des consommations de format standard. Le nombre total de calories consommées pourrait être sousestimé si le format des consommations est plus grand que le format standard. Par exemple, une chopine de bière ordinaire peut être considérée comme un verre standard par les répondants, alors qu'elle équivaut en réalité à 1,66 verre standard. Des renseignements supplémentaires sur les types de boissons alcoolisées les plus couramment consommées permettraient 
FIGURE 2

Projections du nombre de calories consommées sur une période d'un an, sur la base de cinq verres par occasion, selon le type de boisson, chez les élèves de $12^{\mathrm{e}}$ année ayant participé à l'année 2 (2013-2014) de l'étude COMPASS, Ontario et Alberta, Canada
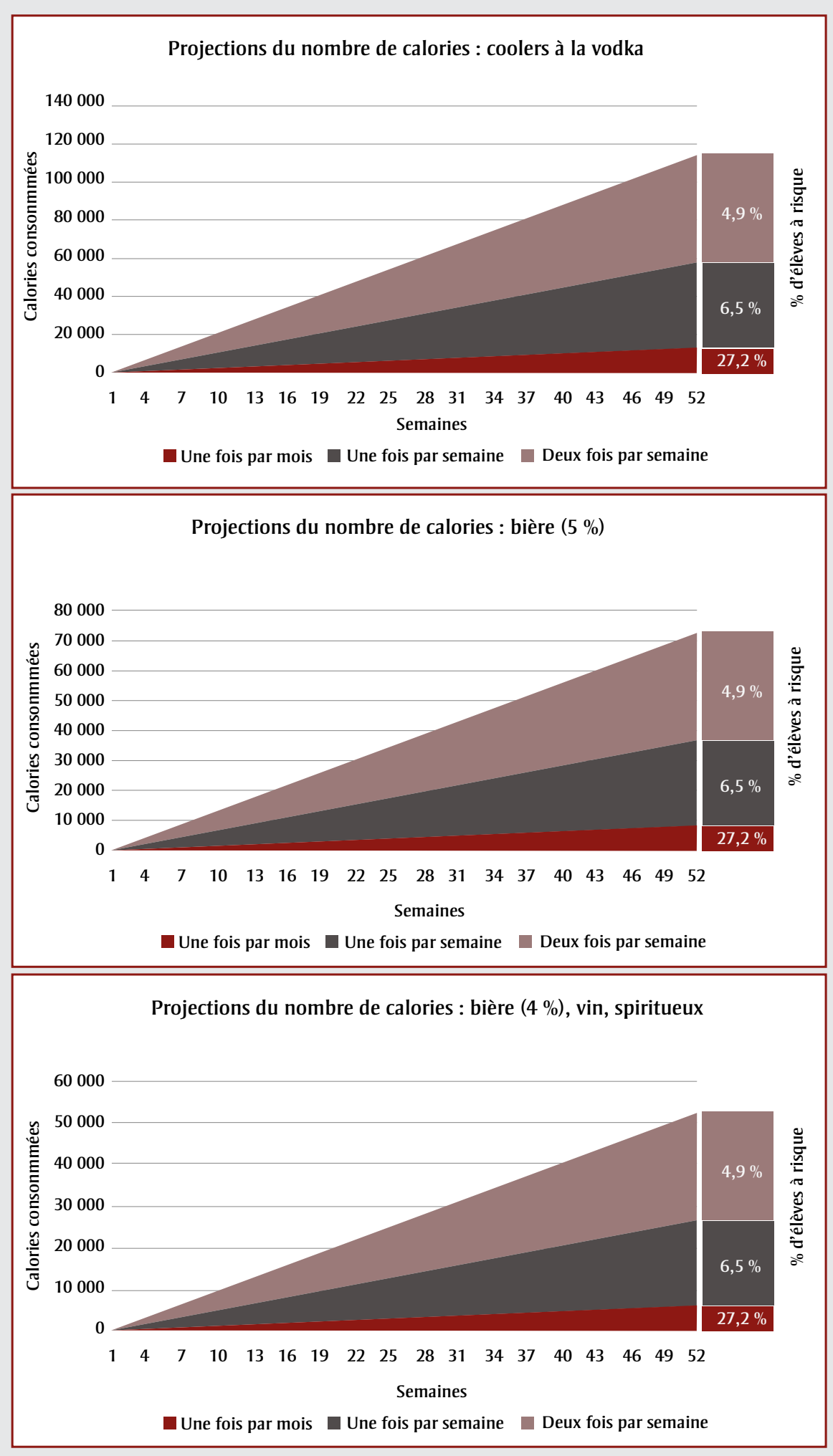

d'obtenir une estimation plus précise de l'apport en calories.

Ces résultats sont fondés sur l'analyse de données secondaires provenant d'un échantillon qui n'est pas représentatif à l'échelle nationale. Ainsi, les habitudes de consommation d'alcool indiquées ici pourraient ne pas être représentatives de l'ensemble des jeunes Canadiens. De plus, comme il s'agit d'une analyse secondaire, les données dont nous disposons pour évaluer la fréquence de consommation d'alcool et la fréquence des abus occasionnels d'alcool ne permettent pas de déterminer avec précision la quantité d'alcool consommée. Pour ce faire, il faudrait disposer de renseignements supplémentaires tels que le nombre de verres habituellement consommés lors d'une même occasion, ainsi que des données plus précises sur la fréquence à laquelle les jeunes consomment de l'alcool, et la fréquence à laquelle ils s'adonnent aux abus occasionnels d'alcool.

Les abus occasionnels d'alcool présentent de nombreux risques pour la santé chez les adolescents $^{31}$. Bien que la présente étude porte expressément sur les calories supplémentaires potentiellement consommées avec l'alcool, la consommation d'alcool chez les personnes d'âge mineur entraîne une multitude d'autres effets négatifs immédiats et à long terme qu'il conviendrait de prendre en compte dans les initiatives de sensibilisation et les interventions en santé publique.

\section{Conclusion}

En dépit des limites abordées plus haut, la présente analyse met en évidence la nécessité, pour les chercheurs et les intervenants, d'accorder davantage d'attention à la consommation d'alcool comme facteur de risque modifiable de l'embonpoint et de l'obésité chez les jeunes. Étant donné qu'une large proportion des jeunes de la population étudiée a déclaré s'adonner fréquemment à la consommation d'alcool et aux abus occasionnels d'alcool, et que les boissons alcoolisées contiennent un nombre élevé de calories, tout indique que l'alcool pourrait être un élément clé à ne pas négliger dans les discussions sur l'obésité.

\section{Remerciements}

L'étude COMPASS a reçu le soutien d'une subvention transitoire de l'Institut de la nutrition, du métabolisme et du diabète des Instituts de recherche en santé du 
Canada (IRSC), grâce à l'attribution du financement prioritaire « Obesity-Interventions to Prevent or Treat » (Interventions pour prévenir ou traiter l'obésité) (OOP-110788; subvention accordée à S. T. Leatherdale) et d'une subvention de fonctionnement de l'Institut de la santé publique et des populations des IRSC (MOP-114875; subvention accordée à S. T. Leatherdale). Le Dr Leatherdale est titulaire d'une chaire de recherche appliquée en santé publique qui est financée par l'Agence de la santé publique du Canada (ASPC) en partenariat avec l'Institut des neurosciences, de la santé mentale et des toxicomanies (INSMT) et l'ISPP des IRSC.

\section{Conflits d'intérêts}

Les auteurs n'ont aucun conflit d'intérêts à déclarer en ce qui concerne ces travaux.

\section{Contribution des auteurs}

KB a effectué les analyses, interprété les résultats, rédigé la première ébauche de l'introduction et de la méthodologie, et collaboré à l'ébauche de certaines parties de l'analyse du présent document. SL a conçu l'idée du document, dirigé l'étude de référence COMPASS, rédigé certaines parties de l'analyse, et révisé toutes les parties du document.

\section{Références}

1. US Department of Health and Human Services. The Surgeon General's vision for a healthy and fit nation. Rockville (MD) : US Department of Health and Human Services, Office of the Surgeon General; 2010. 21 p.

2. Roberts KC, Shields M, de Groh M, et al. L'embonpoint et l'obésité chez les enfants et les adolescents : résultats de l'Enquête canadienne sur les mesures de la santé de 2009 à 2011. Rapports sur la santé. 2012; 23(3):39-43.

3. Tremblay M, Shields M, Laviolette M, et al. Condition physique des enfants et des jeunes au Canada : résultats de l'Enquête canadienne sur les mesures de la santé de 2007-2009. Rapports sur la santé. 2010; 21(1):7-22.

4. World Health Organization. Populationbased approaches to obesity prevention. Geneva $(\mathrm{CH})$ : World Health Organization Library, 2012. 54 p.
5. Agence de protection et de promotion de la santé de l'Ontario (Santé publique Ontario). Obésité chez les enfants et les jeunes : données probantes pour guider l'action en Ontario. Toronto (Ont.), Imprimeur de la Reine pour l'Ontario; 2013. 27 p.

6. Davis MM, Gance-Cleveland B, Hassink S, et al. Recommendations for prevention of childhood obesity. Pediatrics. 2007;120(Suppl 4):S229-S253.

7. Peirson L, Fitzpatrick-Lewis D, Morrison $\mathrm{K}$, et al. Prevention of overweight and obesity in children and youth: a systematic review and meta-analysis. CMAJ Open. 2015; 3(1):E23-33. doi: 10.9778/cmajo.20140053.

8. Malik V, Schulze M, Hu F. Intake of sugar-sweetened beverages and weight gain: a systematic review. Am J Clin Nutr. 2006;84(2):274-288.

9. Ogilvie KK, Eggleton A. L’obésité au Canada : une approche pansociétale pour un Canada en meilleure santé. Rapport du Comité sénatorial permanent des Affaires sociales, des sciences et de la technologie. Ottawa (Ont.), gouvernement du Canada; 2016. 61 p.

10. Berkey C, Rockett H, Colditz G. Weight gain in older adolescent females: the internet, sleep, coffee and alcohol. J Pediatr. 2008;153(5):635-639.e1.

11. Croezen S, Visscher T, ter Bogt, $\mathrm{N}$, Veling M, Haveman-Nies A. Skipping breakfast, alcohol consumption and physical inactivity as risk factors for overweight and obesity in adolescents: results of the E-MOVO project. Eur J Clin Nutr. 2009;63:405-412.

12. Farhat T, Iannotti R, Simons-Morton B. Overweight, obesity, youth and healthrisk behaviors. Am J Prev Med. 2010; 38(3):258-267.

13. Vagstrand K, Barkeling B, Forslund H et al. Eating habits in relation to body fatness and gender in adolescentsresults from the 'SWEDES' study. Eur J Clin Nutr. 2007;61(4):517-525.

14. Suter P, Häsler E, Vetter W. Effects of alcohol on energy metabolism and body weight regulation: is alcohol a risk factor for obesity? Nutr Rev. 1997; 55(5):157-171.
15. Yeomans M. Alcohol, appetite and energy balance: is alcohol intake a risk factor for obesity? Phys Behav. 2010; 100(1):82-89.

16. Piran N, Robinson SR. Associations between disordered eating behaviours and licit and illicit substance use and abuse in a university sample. Addict Behav. 2006;31(10):1761-1775.

17. Leatherdale ST, Rynard V. A cross-sectional examination of modifiable risk factors for chronic disease among a nationally representative sample of youth: are Canadian students graduating high school with a failing grade for health? BMC Public Health [Internet]. 2013 [consulté le 27 février 2016]; 13:569. En ligne à : http://dx.doi.org /10.1186/1471-2458-13-569

18. Fan AZ, Russell M, Stranges S, Dorn J, Trevisan, M. Association of lifetime alcohol drinking trajectories with cardiometabolic risk. J Clin Endocrinol Metab. 2008;93(1):154-161.

19. Leatherdale ST, Brown KS, Carson V, et al. The COMPASS study: a longitudinal hierarchical research platform for evaluating natural experiments related to changes in school-level programs, policies and built environment resources. BMC Public Health [Internet]. 2014 [consulté le 27 février 2016];14: 331. En ligne à : http://dx.doi.org /10.1186/1471-2458-14-331

20. Elton-Marshall T, Leatherdale ST, Manske SR, Wong K, Ahmed R, Burkhalter R. Méthodologie de l'Enquête sur le tabagisme chez les jeunes (ETJ). Maladies chroniques et blessures au Canada. 2011; 32(1):53-61.

21. Centre canadien de lutte contre les toxicomanies. Résumé lié aux Directives de consommation d'alcool à faible risque - Les jeunes et l'alcool [Internet]. Comité consultatif sur la Stratégie nationale sur l'alcool. Ottawa (Ont.), Centre canadien de lutte contre les toxicomanies; 2014. En ligne à : http:// www.ccsa.ca/Resource \% 20Library /CCSA-Youth-and-Alcohol-Summary -2014-fr.pdf

22. Fichier canadien sur les éléments nutritifs (FCÉN) [Internet]. Ottawa (Ont.), Santé Canada; 2015. En ligne à : http:// www.hc-sc.gc.ca/fn-an/nutrition /fiche-nutri-data/cnf_downloads -telechargement_fcen-fra.php 
23. Centers for Disease Control and Prevention. Healthy weight: finding a balance [Internet]. Atlanta (GA) : Centers for Disease Control and Prevention; 2015. En ligne à : https:// www.cdc.gov/healthyweight/calories/

24. Must A, Strauss RS. Risks and consequences of childhood and adolescent obesity. Int J Obes Relat Metab Disord. 1999;23(Suppl 2):S2-S11.

25. deRuiter W, Cairney J, Leatherdale S, Faulkner G. A longitudinal examination of the interrelationship of multiple health behaviours. Am J Prev Med. 2014;47(3):283-289.

26. deRuiter W, Cairney J, Leatherdale S, Faulkner G. The period prevalence of risk behaviour co-occurrence among Canadians. Prev Med. 2016;85:11-16.

27. Boak A, Hamilton HA, Adlaf EM, Mann RE. Drug use among Ontario students, 1977-2015: detailed OSDUHS findings (CAMH Research Document Series No. 41). Toronto (ON) : Centre for Addiction and Mental Health; 2015. 52 p.

28. Patrick ME. A call for research on high-intensity alcohol use. Alcohol Clin Exp Res. 2016;40:256-259.

29. Herciu AC, Laxer RE, Cole A, Leatherdale ST. A cross-sectional study examining factors associated with youth binge drinking in the COMPASS study: Year 1 data. J Alcohol Drug Depend [Internet]. 2014; 2:172. En ligne à : http://dx.doi.org /10.4172/2329-6488.1000172

30. National Institute on Alcohol Abuse and Alcoholism (NIAAA). Alcohol calorie calculator [Internet]. Bethesda (MD) : NIAAA [date inconnue; consulté le 1er mars 2016]. En ligne à : http://rethinkingdrinking.niaaa.nih .gov/Tools/Calculators/Calorie -Calculator.aspx

31. Miller J, Naimi T, Brewer R, Everett Jones S. Binge drinking and associated health risk behaviors among high school students. Pediatrics. 2007; 119(1):76-85. 